\section{Microwave Intensified Synthesis: Batch and Flow Chemistry}

\author{
Chi Ching Lau, ${ }^{[a] 1}$ Mustafa Kemal \\ Bayazit, ${ }^{[a] 1}$ Philip James Thomas \\ Reardon, ${ }^{[b] 1}$ and Junwang Tang ${ }^{*[a]}$
}

Abstract: Many studies have been conducted on organic and inorganic synthesis by microwave heating owing to its special heating mechanism, leading to improved reaction rate, higher purity and yields. We specifically demonstrated microwave heating in the fabrication of nanoparticles and polyester. By finetuning the microwave and experimental parameters, the materials prepared have shown excellent physical and bio-properties, e.g. narrow particle size distribution, controlled morphology, varied molecular structure and so forth. We further highlight the recent procedure of using fluidic reactors on preparing the nanoparticles. The experimental design strategies and fundamentals of the microwave interaction with chemicals are presented. Furthermore, the issues facing in this area are also discussed.

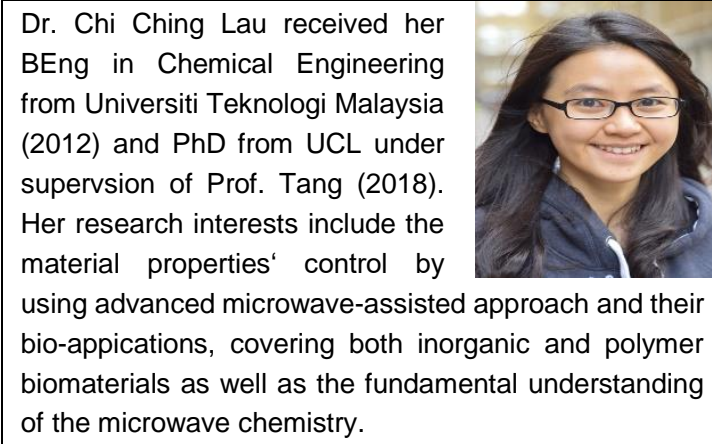

[a] Chi Ching Lau, Dr Mustafa Kemal Bayazit, Prof Junwang Tang* Department of Chemical Engineering

University College London

Torrington Place

WC1E 7JE

E-mail: junwang.tang@ucl.ac.uk

[b] Dr Philip James Thomas Reardon

Graduate School of Engineering

Nagoya Institute of Technology

Gokisocho, Showa Ward

Nagoya

466-8555
Dr. Mustafa K. Bayazit received his MSc in Chemistry from Abant Izzet Baysal University before receiving his $\mathrm{PhD}$ at the University of Durham in 2010. He then worked at ICL as a PDRA for 4 years. Following that, he joined the group of Prof. Tang and has been here for three years as a senior PDRA. His current work involves the processing and applications of 2D nanomaterials and microwave intensified fluidic systems. He is the recipient of doctoral fellowship awarded by the Scientific and Technological Research Council of Turkey, the 1st Community Achievement Award by the Centre for Turkey Studies and Development in 2012, and was listed in Marquis Who's Who in the World 2013.

Dr. Philip J. T. Reardon received his MSc in 2010, and PhD under supervision of Prof. Tang in 2015 from University College London (UCL), UK. He was the recipient of a doctoral grant from the Engineering and Physical Sciences Research Council. After working as a PDRA at the Eastman Dental Institute (UCL) he was honoured with a Research Fellowship from Japan Society for the Promotion of Science in 2017, and moved to Nagoya Institute of Technology, Japan. His research interests include the engineering of nanomaterials and their application in tissue engineering and drug delivery.

Prof. Junwang Tang is a Fellow of RSC, the Director of UCL Materials Hub and Professor of Chemistry and Materials Engineering in the Department of Chemical Engineering at University College London. He received his $\mathrm{PhD}$ in Physical Chemistry from Dalian Institute of Chemical Physics, China in 2001. After that, he was appointed as a JSPS Fellow at NIMS, Japan and a senior researcher in the Department of Chemistry at Imperial College London, concentrating on solar fuels synthesis and the underlying research mechanism by time-resolved spectroscopies. He then took a faculty position at UCL in 2009. His current research interests lie in photocatalytic small molecule activation (eg. $\mathrm{CH}_{4}, \mathrm{~N}_{2}, \mathrm{H}_{2} \mathrm{O}, \mathrm{CO}_{2}$ ) and mechanistic aspects of photocatalysis and water treatment, alongside microwave intensified fluidic chemistry. 


\section{Introduction Chemistry \\ to Microwave}

Microwaves, which forms part of the electromagnetic spectrum, have a wavelength from $1 \mathrm{~mm}$ to $1 \mathrm{~m}$ and a frequency of $300 \mathrm{MHz}$ to $300 \mathrm{GHz}$. In a standard system they are generated by a magnetron before being released into a chamber, which is covered by a protection layer to avoid leaking of microwave irradiation to the surroundings. After the introduction of microwaves in chemical reactions in 1986 [1, 2], a significant number of publications regarding the microwave-assisted synthesis of organic and inorganic materials in batch and continuous-flow reactors have been reported.[3, 4] Today, well-developed commercial microwave reaction systems provide advanced monitoring and control of reaction variables during synthesis, allowing high reproducibility and reduced safety concerns.

In a microwave reaction system, the mechanism of heat generation is known as dielectric heating, the two major mechaisms of which are dipolar polarisation and ionic conduction. In the former, microwave electromagnetic waves cause molecular rotation, enabling internal heat generation due to fricition. [5]

The ability of a substance or a solvent to absorb microwave energy is dependant on the dielectric constant, dielectric loss and loss tangent. The loss tangent is defined as the efficiency of the solvent to convert the microwave energy into thermal energy, which is the ratio of dielectric loss (coupling efficiency of the solvent with the microwave) to the dielectric constant (ability to store electric charges). The higher the dielectric loss, the faster the solvent reaches its pre-set temperature. The solvents are categorised into three groups: high (dielectric loss $>14$ ), medium (dielectric loss 1-13.99) and low (dielectric loss <1) microwave absorbance..$^{[1]}$ Importantly, the dielectric constant alone does not represent the efficacy of a material for microwave absorbance. For instance, water with high dielectric constant is classified as having a medium microwave absorbance owing to its lower loss tangent and dielectric loss. Molecular structure of a solvent also plays a key role in absorbing microwave irradition; short chain alcohols are heated faster than long chain hydrocarbons to similar temperatures. This is because hydrocarbons with a longer backbone inhibit microwave heating, due to their reduced ability to align themselves with the changing electric field.[6, 7]

Table 1 Dielectric constant, loss tangent and ielectric loss for solvents (measured at room temperature and $2450 \mathrm{MHz}) \cdot[5]$

\begin{tabular}{|c|c|c|c|}
\hline Solvent & $\begin{array}{l}\text { Dielectric } \\
\text { Loss }\end{array}$ & $\begin{array}{l}\text { Dielectric } \\
\text { Constant }\end{array}$ & $\begin{array}{c}\text { Loss } \\
\text { Tangent }\end{array}$ \\
\hline \multicolumn{4}{|c|}{ Strong microwave absorbance } \\
\hline Ethylene Glycol & 49.950 & 37.0 & 1.350 \\
\hline Formic Acid & 42.237 & 58.5 & 0.722 \\
\hline Ethanol & 22.866 & 24.3 & 0.941 \\
\hline Methanol & 21.483 & 32.6 & 0.659 \\
\hline 1-Propanol & 15.216 & 20.1 & 0.757 \\
\hline \multicolumn{4}{|c|}{ Medium microwave absorance } \\
\hline Water & 9.889 & 80.4 & 0.123 \\
\hline 1-Butanol & 9.764 & 17.1 & 0.571 \\
\hline Isobutanol & 8.248 & 15.8 & 0.522 \\
\hline Acetone & 1.118 & 20.7 & 0.054 \\
\hline Acetic Acid & 1.079 & 6.2 & 0.174 \\
\hline
\end{tabular}

\begin{tabular}{|l|c|c|c|}
\hline \multicolumn{4}{|l|}{ Weak microwave absorbance } \\
\hline Chloroform & 0.437 & 4.8 & 0.091 \\
\hline $\begin{array}{l}\text { Dicholomethan } \\
\text { e }\end{array}$ & 0.382 & 9.1 & 0.042 \\
\hline $\begin{array}{l}\text { Tetrahydrofura } \\
\mathrm{n}\end{array}$ & 0.348 & 7.4 & 0.047 \\
\hline Toluene & 0.096 & 2.4 & 0.040 \\
\hline Hexane & 0.038 & 1.9 & 0.020 \\
\hline
\end{tabular}

Table 2 Dielectric materials chart $[6,8]$

\begin{tabular}{|l|c|c|}
\hline \multicolumn{1}{|c|}{ Material } & $\begin{array}{c}\text {-Loss } \\
\text { Tangent } \\
\times \mathbf{1 0}^{-4}\end{array}$ & $\begin{array}{c}\text { Melting } \\
\text { Point }\end{array}$ \\
\hline C \\
\hline TEFLON $^{\mathrm{TM}}$ & $1-2$ & 327 \\
\hline Polyethylene & $1-2$ & $115-135$ \\
\hline Fused Quartz & $1-2$ & Resistant \\
\hline Polystyrene & $2-3$ & 100 -120 \\
\hline Fused Silica & $2-3$ & Resistant \\
\hline Polypropylene & $3-4$ & $130-171$ \\
\hline Boron nitride & $3-4$ & Resistant \\
\hline Sapphire & $3-4$ & Resistant \\
\hline Mica & $3-4$ & over 700 \\
\hline Aluminum oxide & $6-7$ & Resistant \\
\hline Butyl rubber (uncured) & $9-10$ & melt \\
\hline Borosilicate glass & $10-20$ & Resistant \\
\hline Steatite & $10-20$ & Resistant \\
\hline Natural Rubber (uncured) & $20-50$ & melt \\
\hline Partially fluorinated polyethylene & $20-50$ & 210 \\
\hline Styrene-Butadiene rubber (25/75) & $20-50$ & 120 \\
\hline Wollastonite & $20-50$ & 1540 \\
\hline Pyrex ${ }^{\text {TM }}$ Glass & $50-100$ & Resistant \\
\hline Polycarbonates & $50-100$ & 155 \\
\hline Nitrile rubber & $100-500$ & \\
\hline Nylon ${ }^{\mathrm{TM}}$ & $100-500$ & 190 to 350 \\
\hline Epoxy & $100-500$ & Resistant \\
\hline Silicone Rubber & $100-500$ & max 300 \\
\hline Soda-lime Glass & $100-500$ & Resistant \\
\hline Water & 1230 & \\
\hline
\end{tabular}

Understanding the mechanism of microwave heating, in particular the degree of microwave coupling and hence heating efficacy of each reactant species, is highly important. The material properties can be fine-tailored by optimising the microwave and experimental parameters. In designing a microwave-assisted chemical experiment, there are four key factors that need to be considered: i) the loss tangent of the solvent species (Table 1), ii) the microwave absorption capacity of the reaction vessel (Table 2), iii) the accuracy of temperature measurement, and iv) mass transfer.

In addition, the selection of microwave mode (i.e. singlemode vs multi-mode) in preparing a material is also crucial. A single-mode microwave system is usually preferable for a reaction requiring high microwave intensity, and a multi-mode system is usually used for a 
large scale reactor requiring relatively homogeneous low microwave intensity.[9].[10] Furthermore, the position of a reactor in the single-mode microwave is important, which will affect the reproducibility of a chemical process, which will be discussed later.

In short, this personal account aims to present the group's experience in utilising a microwave-assisted approach for preparing nanoparticles and polyesters, whilst conveying the current state of the art knowledge in this field. Furthermore, based upon recent developments in this laboratory of a well-controlled microwave intensified fluidic system to synthesise nanoparticles, influence of batch and fluidic systems on microwave-assisted materials synthesis will be discussed.

\section{Microwave Batch System for Synthesis of Nanoparticles}

The unique properties of nanophase materials, originating from their size, morphology, and porosity, indicate that controlling these parameters is of the highest importance in the field of nanomaterials synthesis. A number of different fabrication methods for nanomaterials have been developed, in particular wet chemical techniques which can produce nanomaterials with different morphologies have been widely reported. However, precise control of nanomaterials parameters by a scalable production approach that are rapid and efficient, still remain a challenge in the commercialisation of nanosized materails.

\subsection{Experimental design and motivation}

Compared to conventional wet chemical techniques, the implementation of microwave irradiation (MI) to hydrothermal/solvothermal synthesis is particularly attractive for nanomaterial synthesis due to its characteristic rapid, volumetric and selective heating. In this technique, solvents (e.g. water specifically in the case of hydrothermal condition) are brought to temperatures above their boiling points, achieving an autogenous pressure through heating in a sealed container. It offers many advantages, including greater precursor reactivity, energy efficiency, and more importantly potential formation of distinct metastable phases.[11] Control of reaction conditions is critical for dictating the stability of each species involved in the reaction, and importantly, it is achievalble to synthesise different metastable, kinetically controlled morphologies and phases. In comparison reaction control using conventional solvothermal technique is frequently deficient, due to the non-selective and indirect nature of the heating. Therefore, the employment of the novel heating technique $\mathrm{MI}$, which allows excellent control of reaction parameters, offers great potential to nanomaterial synthesis when applied to batch solvothermal synthesis.

The potential of $\mathrm{Ml}$ for enhancements in controllable batch nanomaterial synthesis has only recently gained interest, with the realisation of more regulated nanocrystal synthesis and improved quality and yield when compared to conventional heating methods. This can be attributed at least in part to recently developed microwave reaction systems, which allow measurement and control of reaction conditions (e.g. temperature and pressure) more accurate and reproducible.[12]

Conventional heating methodologies for batch hydrothermal/solvothermal synthesis typically involve placing a sealed reaction vessel containing the reactants in a convection oven, whereby the temperature is controlled by the set temperature of the oven. In microwave-assisted synthesis system, the sealed reaction chamber is placed inside a Ml chamber, in which the temperature of the reaction is tuned by varying the microwave power with a feed-back loop to achieve accurate temperature measurement and control. This microwave irraidation offers direct heating and rapid heating rates. A specially designed commercial reaction system is utilised in the authors studies, allowing hydrothermal/solvothermal synthesis (above room temperature and $1 \mathrm{~atm}$ pressure) to be accurately controlled using feedback from the measured temperature of the reaction vessel via fiber optic temperature measurement (Figure 1), thus enabling reliable control during synthesis of nanomaterials. In addition to excellent control of reaction temperature, the latest microwave-assisted methods reportedly possess such advantages as rapid heating, increased reaction rate and excellent reproducibility, resulting in high energy efficiency, narrow particle distribution, high purity and high yields,. [3, 4, 12-14]

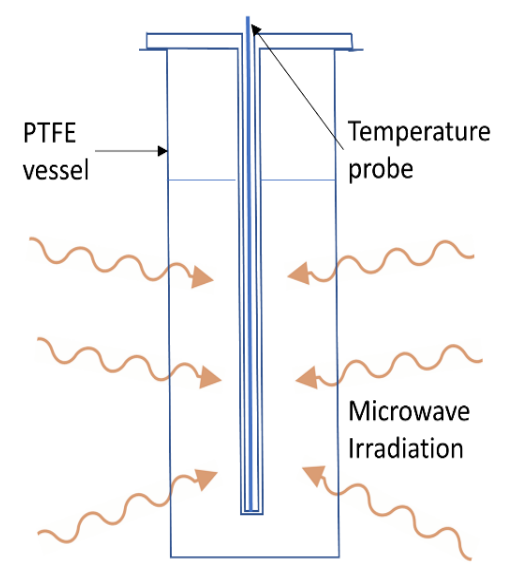

Figure 1. Schematic of microwave reaction vessel.

\subsection{Effects of microwave heating on nanomaterials synthesis}

With the introduction of $\mathrm{MI}$ to chemical synthesis, there have been numerous reports about the exact influence of this technology on nanomaterial synthesis. It can be argued that the majority of microwave dielectric heating phenomena can be explained by purely kinetic/thermal effects, arising from the rapid heating rates and higher bulk reaction temperatures attainable with $\mathrm{Ml}$ assisted synthesis. This is particularly pertinent to the use of solvents or precursor solutions with a high value of tan $\delta$, (see table 1) which may become rapidly "superheated" far above their boiling points when irradiated in a sealed vessel (as in microwave assisted solvothermal/hydrothermal synthesis). Such temperature profiles are practically very difficult to 
reproduce with conventionally equipment, making direct comparison limited. These differences in temperature profiles even if moderate can have a dramatic effect on reaction kinetics, potentially changing reaction outcomes.

It has been posited that the efficient and uniform "in core" heating associated with MI, with minimised thermal gradients should result in more rapid and spatially uniform nucleation and growth of nanophase crystals.[15] An argument based on these changes to nucleation and growth processes can then be used to explain the smaller sizes and size distribution frequently observed in microwave enhanced synthesis in comparison to conventional heating. When using conventional methods, the reaction system is heated by thermal conduction through a container wall, then convection via agitation; hence the temperature distribution in the bulk solution will be inhomogeneous in comparison to the homogeneous heating achieved by $\mathrm{Ml}$ heating. The processes of nucleation and growth depend on local temperature distribution of the system, so inhomogeneity or homogeneity in temperature distribution should cause either a broad or narrow distribution in particle size and shape, respectively. A further argument can be made based on nucleation and growth processes in relation to explaining smaller particle size. The relatively faster heating rates associated with $\mathrm{Ml}$ compared to conventional heating allows an increased nucleation rate and relatively higher consumption of monomer units, in general resulting in crystallisation of more smaller particles, even if the subsequent growth is fairly rapid as the monomer amount left for growth will be relatively low. This observation was confirmed in a study comparing conventionally heated and $\mathrm{MI}$ heated hydrothermal methodologies for the production of microporous material.[16] Furthermore, it was proposed that the specific heating that is characteric of MI can allow the production of "hot spots" when strongly coupled solvents are employed, that would lead to faster nanocrystal development and high yield.[12]

In recent years, there has been a large amount of interest in the use of nanomaterials for biomedical applications, given the unique advantages afforded by these materials. For example, it is reported that they can be excellent platforms for tissue regeneration and drug transportation.[17, 18] Microwave heating produces homogeneous (molecular level) heating of polar solution systems, achieving fast nucleation and growth mechanisms which is pererential for preparation of nanophase biomedical materials. However, the application of this technology has not been extensively investigated in the biomaterials field. Herein, our new results on biomedical nanomaterials synthesis and key factors controlling their morphology, phase and particle size are presented in detail.

Within this field, the potential for using $\mathrm{Ml}$ for calcium phosphate $(\mathrm{CaP})$ biomaterials' preparation was initially demonstrated by the synthesis of hydroxyapatite and tricalcium phosphate particles.[19, 20]Recently, motivated by the potential of this technology, we developed a novel microwave-assisted method to efficiently produce $\mathrm{CaP}$ nanoparticles with controlled size and chemical composition. During a systematic comparison with convection heated hydrothermal synthesis, we found that microwave synthesis produces nanomaterials with about $50 \%$ higher yields and reduced variation in particle size and morphology.
Furthermore, reaction time of only 1 minute was required compared with 20 minutes in conventional heating .[21]

Mesoporous structured calcium phosphates were previously achieved through template-directed growth methods, such as using surfactants.[22, 23] However, the control over particle distribution, morphology and size of these materials was not ideal, and there remains concerns that using templating materials may result in unwanted organic residues being incorporated into the final products. We found that microwave synthesis produces nanomaterials with about 1.7 times higher surface area and 5 times higher pore volume than conventionally heated materials respectively, a critical parameter for biomedical materials, in particular regarding drug delivery systems.[21, 24]. Furthermore, no additional structure-directing media were used in our methodology. Next we investigated the relationship between the $\mathrm{Ml}$ experimental parameters and the properties of the prepared materials.

\subsection{Effect of experimental parameters on the nanoparticles properties}

Reaction kinetics are influenced by the microwave intensity and the reaction precursors ' composition. The final size, morphology, and quality of the microwavegenerated materials were found to depend on the choice of reactants, the applied power, reaction time, and temperature.

In our studies, we demonstrated that reaction temperature could be used to control the morphology of $\mathrm{CaP}$ nanomaterials. As can be seen from Figure 2 , when a reaction temperature of $60^{\circ} \mathrm{C}$ was used mesoporous nanorods were produced, when the temperature was increased (up to $200^{\circ} \mathrm{C}$ ) mesoporous nanoplates of $\mathrm{CaP}$ were synthesised.[25] The greater temperature control afforded by the direct coupling of $\mathrm{MI}$ with solvent, allow different metastable morphologies prepared in this case. Importantly, the different shaped mesoporous CaP nanomaterials exhibited variation in the loading and release of therapeutic molecules. For example, the nanoplates exhibited 4 times higher protein loading than the nanorods. [25] Therefore, these nanostructured particles have strong potential to act as controlled drug delivery systems.

Interestingly, MI time was found to control the porosity of CaP materials but not morphology. Control over protein loading was demonstrated by increasing mesopore volume. Increased bovine serum albumin (a common standard protein) loading was observed with increased porosity for the mesoporous nanoplate materials, equivalent to ca. $40 \%$ increase in loading wt \% from materials using a $1 \mathrm{~min}$ Ml reaction time (pore volume $-0.1 \mathrm{~cm}^{3} \mathrm{~g}^{-1}$ ) to $5 \mathrm{Ml}$ minute reaction time (pore volume $-0.25 \mathrm{~cm}^{3} \mathrm{~g}^{-1}$ ), suggesting a direct correlation between $\mathrm{Ml}$ eaction time, mesoporosity and protein loading. [21] 


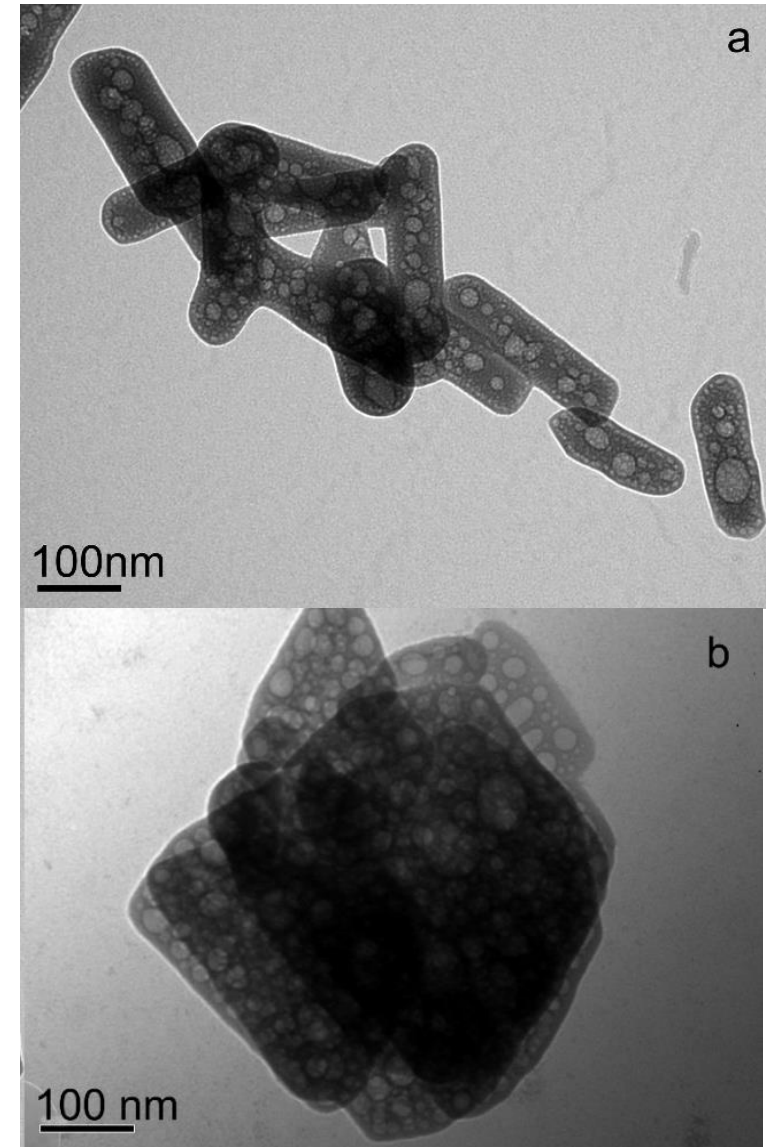

Figure 2 CaP (a) nanorods and (b) nanoplates synthesised by microwave intensified hydrothermal technique at (a) $60{ }^{\circ} \mathrm{C}$ and (b) $200{ }^{\circ} \mathrm{C}$. Modified with permission from AHM.[25]

As introduced, mesoporous $\mathrm{CaP}$ materials were previously reported using additional surfactant materials, however addition structure directing agents were not employed in our methodology. Our studies suggested that the organic solvent can also function as a structure directing agent, creating pores when the material forms around the solvent molecules or indeed with the inclusion of water. Small pores were then produced upon solvent removal, and then small particles quickly agglomerate under high energy conditions, forming larger mesopores. Furthermore, the number of pores forms in the nanomaterials produced by conventionally heated solvothermal synthesis was lower relative to that produced by $\mathrm{Ml}$ intensified synthesis. $\mathrm{Ml}$ is characterised by the direct coupling of irradiation with solvent molecules, producing rapid and controlled volumetric heating with high local temperatures, allowing for effective and uniform pore production. Therefore it can be concluded that formation of mesoporous $\mathrm{CaP}$ are controlled by both the solvent and heating methodology, with this process posited to take place to a larger extent in the materials produced at higher temperatures and using MW intensified synthesis.

Another critical reaction parameter in the microwaveassisted synthesis of nanomaterials is selection of the reaction solvent which can not only change porosity as mentioned above but also phase of nanoparticles. For the first time, by utilising a microwave-assisted synthetic method, we were able to produce three commonly employed CaP phases ( $\beta$-TCP. HA, and dicalcium phosphate anhydrous) in a rapid and efficient manner and under mild conditions, by only varying the reaction solvent.[26] MI heating in a closed vessel system produces a high vapor pressure when volatile solvents are used. Methanol and ethanol used in this study have relatively high vapor pressure of 400-550 psi and 300400 psi respectively. It was found that vapour pressure had a contribution in combination with microwave heating to accelerate the reaction rates. As we found that DCPA and HA could also be synthesised at room temperature in similar solvents but with a very long reaction times, we believe Ml greatly increased the reaction rates and product yields. Importantly, synthesis of the important resorbable bioceramic $\beta$-TCP typically requires high temperature aqueous conditions ( $>$ $800{ }^{\circ} \mathrm{C}$ ), or long reaction times of approximately eight hours in methanol, but can be synthesised in 5 minutes using the Ml heating technique. Therefore, the rapid synthesis and phase selectivity of $\mathrm{CaP}$ biomaterials were attributed to a highly pressurised system and MI heating.

Due to the progress in the controlled synthesis of $\mathrm{CaP}$ nanomaterials utilising appropriate reaction solvents, the feasibility of the microwave-assisted synthesis of biphasic and high aspect ratio $\mathrm{CaP}$ nanobioceramic compounds for drug delivery and bone tissue regeneration was then assessed. Control of CaP nanomaterials morphology and more importantly phase was found by changing the relative quantities of $\mathrm{EtOH}$ used as a co-solvent with $\mathrm{H}_{2} \mathrm{O}$. When a $\mathrm{H}_{2} \mathrm{O} / \mathrm{EtOH}$ volumetric ratio of $80 / 20$ was used we were able to produce high aspect nanowire calcium phosphate materials (aspect ratio of ca. 54, see figure 3), with a biphasic composition of $\mathrm{HA}$ and monetite $(72.0 \%$ monetite, $28.0 \% \mathrm{HA}$ ). The aspect ratio of the nanowires/nanoneedles was also found to be sensitive to changing either the $\mathrm{Ca} / \mathrm{P}$ shoichiometric ratio of the reactants, or by changing the point of $\mathrm{EtOH}$ addition in the reactant mixing procedure. It was observed that varying the $\mathrm{Ca} / \mathrm{P}$ reactant stoichiometry to a higher value of 1.67 increased the relative quantity of HA (up to $91.5 \%$ ) in these 1D biphasic calcium phosphate materials. This was further achieved by increasing the reaction $\mathrm{pH}$ to 10 , implying that the composition of the nanowires/nanoneedles can be controlled using either of these variables.[27]

Conventionally, control over the shape of inorganic nanoparticles is acheived through the application of surfactants or chelating agents. However, in our reaction system, rapid energy input and high-pressure conditions fostered through microwave heating produced 2D and 1D CaP nanoparticles when a solvent composed of high $\mathrm{H}_{2} \mathrm{O} / \mathrm{EtOH}$ volumetric ratio was employed. Previously, Peng et al. reported that CdSe nanocrystals underwent diffusion-controlled 1D growth when there was a high chemical potential of monomer (precursor species) in the solution.[28] Similarly, one dimensional hydrothermal growth of HA nanowires were described by Costa et al., and attributed to a diffusioncontrolled HA crystal shape evolution mechanism based upon varying chemical potential of solution. Therefore, it can be inferred that the key factors for orientated growth are a high chemical-potential environment and an anisotropic crystal structure.

In our studies, a similar theory can be used to explain the observed $\mathrm{CaP}$ nanocrystal shape evolution (Figure $3)$. In this case, the size and aspect ratio of the materials 
are dependant upon the change of solvent composition $\left(\mathrm{EtOH} / \mathrm{H}_{2} \mathrm{O}\right.$ ratio) using microwave heating, to the concentration of solute species $\left(\left[\mathrm{Ca}^{2+}\right]\right.$ and $\left.\left[\mathrm{HPO}_{4}{ }^{2-}\right]\right)$ in the crystal growth phase. The concentration of solute species remaining in solution after the nucleation phase is governed by the number of nuclei formed, with a relatively small number of nuclei desirable for unidirectional growth. It has been reported that addition of ethanol to an aqueous solution can lead to disruption of the bonding in the water structure and with calcium cations, lowering the energy barrier to deaquation and hence facilitating nucleation of the most stable calcium phosphate species.[29] Furthermore, the relatively higher dieclectric loss of $\mathrm{EtOH}$ than water (stronger interaction between $\mathrm{EtOH}$ and microwave radiation) means that the addition of EtOH should increase the dielectric loss (heating effect) of an aqueous medium, and thus induce rapid formation of $\mathrm{CaP}$ nuclei by promptly weakening the interaction between the ionic reactant species and the hydration shell.

Importantly, microwave heating have been shown to accelerate crystal growth in comparison to conventional heating,[16] and to accelerate Brownian motion, giving faster effective circulation of solute in solution, hence favouring elongated growth. Therefore, it is believed that the anisotropic growth of calcium phosphate nanowires and nanoneedles is governed by the intrinsic chemistry of specific faces, the local solution details, and the mode of foreign energy activation (e.g.microwave assisted-solvothermal heating). In other words, EtOH faciliates 3D growth due to lower concentration of solute ions (higher nucleation) while water favors 1D or 2D dimensinal growth due to lower nucleation and hence higher concerntations of solute species under microwave conditions.

Therefore, it is believed that the anisotropic growth of calcium phosphate nanowires and nanoneedles is governed by the intrinsic chemistry of specific faces, the local solution details, and the mode of foreign energy activation (e.g.microwave assisted-solvothermal heating). In other words, EtOH faciliates 3D growth due to lower concentration of solute ions (higher nucleation) while water favors $1 \mathrm{D}$ or $2 \mathrm{D}$ dimensinal growth due to lower nucleation and hence higher concerntations of solute species under microwave condition.
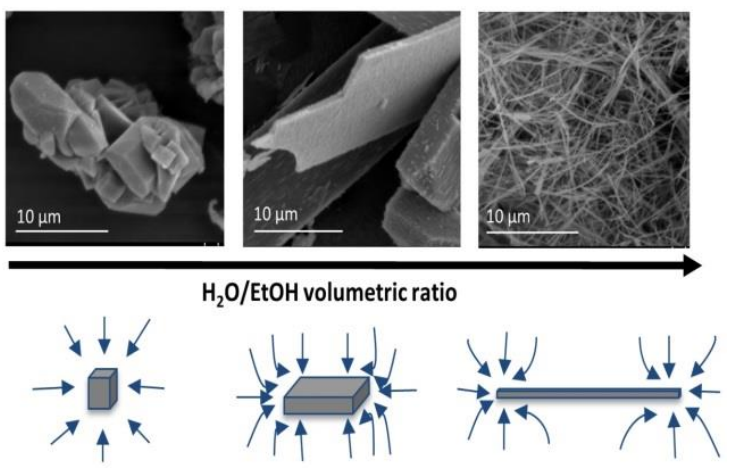

Figure 3 Illustration of the dimensional variation in crystal growth process with different reaction solvent composition, hypothesised to be controlled by governed by the precursors concentration in the bulk, resulting in a transition from either $13 \mathrm{D}$ or to $31 \mathrm{D}$ growth of calcium phosphate when the $\mathrm{H}_{2} \mathrm{O} / \mathrm{EtOH}$ volumetric ratio was increased from 1:4-4:1. Small blue arrows represent precursor flux. Modified from figure published [27]

In summary, our MW-assisted intensified synthetic approach enables the rapid and efficient production of morphology and compositionally controlled $1 \mathrm{D}, 2 \mathrm{D}$ and 3D biomaterials, with the potential for tuneable mechanical and degradation properties in combination with the delivery of therapeutic agents, which have exciting potential for improved graft materials for bone tissue engineering.

\section{Microwave Batch System for Synthesis of Polyesters}

Polyesters, such as poly(glycerol sebacate), poly(lactic acid) and poly (glycolide), are useful polymers that are extensively used in biomedical applications. These degradable polyesters are preferable for bone implants and drug carriers since no additional steps are required to remove them after the surgery.

The use of MI to prepare polyester is highly preferred owing to its selective heating mechanism. Compared to conventional heating methods, MI has the capability to finely tune materials properties while requires shorter reaction time. Furthermore, MI can also improve the product yield and provide great selectivity.[31, 32] This is because the molecular species involved in the polycondensation stage of polyester synthesis are usually highly polar, for example water which is eliminated during the reaction.[33] Additionaly, MI can affect the molecular structure of polyesters produced. For instance, a mixture of cyclic and linear poly(lactic acid) was found when heated with MI.[34]

\subsection{Experimental design and motivation}

In principle, water is eliminated as the side product during the polyesterification process. Thus, purging the reaction medium using an inert gas is very important, as it will help remove water from the medium and prevent the likely unexpected hydrolysis.

Aydin et al reported the preparation of poly(glycerol sebacate) (PGS) in a $650 \mathrm{~W}$ domestic multi-mode microwave (White Westinghouse, USA).[35] In a typical experiment, the monomers of PGS (i.e. sebacic acid and glycerol) were irradiated for 3 minutes with 10 seconds of intervals to obtain the pre-polymer of PGS (pre-PGS). The curing process of the pre-PGS was reduced to 16 hours from 2 to 5 days at $150{ }^{\circ} \mathrm{C}$. Without precisely controlling the real time temperature during polyesterification, the glycerol monomers were found to be evaporated significantly (molar ratio of sebacic acid to glycerol: 78:22) as compared to the initial ratio (100:100), owing to the highly efficient coupling between glycerol molecules and the MI.[35]

$\mathrm{Li}$ et al modified the $\mathrm{MI}$ approach to prepare prePGS.[36] The authors used a 600W domestic microwave (Panasonic NN-ST642W) and measured the temperature of the reaction medium offline every 1 minute using a thermometer. Similar to previous 
procedures, the monomers were irradiated up to 30 minutes with cooling intervals. The esterification of prePGS prepared by microwave for 15 minutes was similar to the one prepared by 6 hours using conventional heating. The authors also quantified the degree of the esterification of the pre-PGS and correlated it with the physical status of the PGS (Figure 4). The PGS changed from a brittle wax to viscous liquid before elastomer formation. However, the on-line temperature was not monitored, which may have affected the evaporation of the monomers and influenced reproducibility. In addition, the reaction medium was not stirred, and this would create a non-uniform temperature distribution during the $\mathrm{MI}$ process.

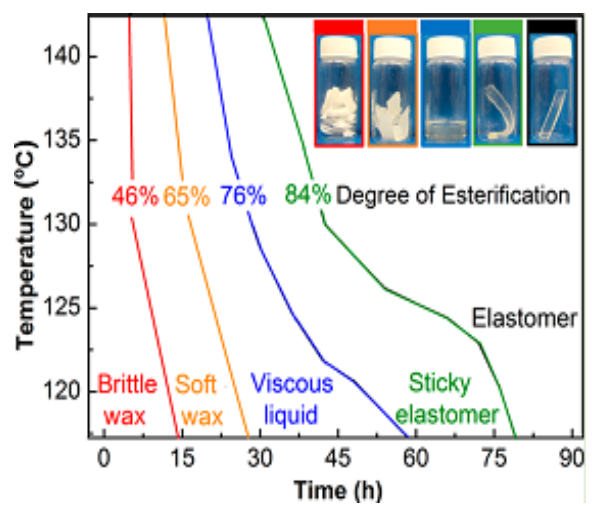

Figure 4 The physical status of the poly(glycerol sebacate) with the corresponded degree of esterification at different reaction temperature. This is adapted with permission from REF.[36], American Chemical Society.

We have recently developed a novel system to tailor the degree of esterification of PGS using a MI method.[37] The degree of esterification was calculated based on the amount of water (side product) collected during the polycondensation process. Our setup (Figure 5) consists of a round bottom flask $(50 \mathrm{~mL})$ made of borosilicate glass containing two PTFE tubes. The first PTFE tube is connected to the inert gas supply and the second one is linked to the Drechsel bottle (solvent or water collection). The PTFE tubes must be tight and completely clean. The outlet of the PTFE tube carrying the inert gas is located lower than the inlet of the collector tube, avoiding the inert gas bypassing the reaction mixture. The PTFE tubes are utilised in this system because it can resist relatively high temperature and can be easily replaced if it is contaminated.

The experiments were conducted in both a single-mode and multi-mode microwave (MARS and Discover, CEM Microwave Technology Ltd.) at 150W (3 minutes per cycle with cooling intervals). The real-time temperature was monitored with a limit of $130{ }^{\circ} \mathrm{C}$ and the reaction medium was stirred vigorously throughout the reaction. After each microwave cycle, the collected water was measured to quantify the degree of the esterification of the polymer. The precursors were well-mixed in the flask before microwave treatment to ensure homogeneous heating. The prepared pre-PGS was then cured in a vacuum oven for $2-48 \mathrm{~h}$ at $120^{\circ} \mathrm{C}$.

When comparing our Ml experimental method with those previously reported, the key improvements are: i) on-line temperature monitoring, ii) relatively homogeneous temperature distirbution, iii) inert gas condition and iV) on-line monitoring of the degree of esterification. The real time temperature control is highly important for improving both the reproducibility and material properties, as the mechanical properties and degradation rate of PGS are dependant upon the ratio of the constituent monomers. Without temperature monitoring, the monomers could evaporate to some extent. In addition, the solid wax of the pre-PGS could block the PTFE tube and inhibit water removal when the system is overheated. In our design, the presence of the toluene with low boiling point $\left(110{ }^{\circ} \mathrm{C}\right)$ reduces the evaporation of the monomers when removing water from the medium. Futhermore, the inert gas is crucial to avoid the oxidation of the monomers. It also helps to remove water during the polycondensation. Lastly, the water collected can be used to tailor the degree of the esterification of the pre-PGS and to estimate the curing time.

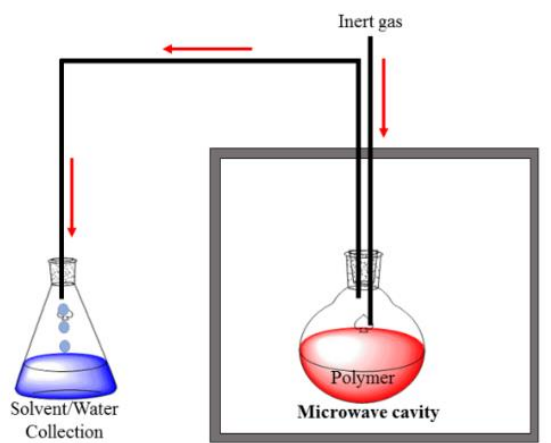

Figure 5 Experimental setup to synthesis the pre-PGS in the single-mode and multi-mode microwave cavity.

As mentioned above, $\mathrm{Ml}$ reduces reaction time in comparison to conventional heating methods.[35-37] As shown in Figure 6, MI also alters the pre-PGS structure owing to the special heating mechanism, leading to a wider range of degradation rates and providing a high degree of flexibility to control the mechanical properties. For instance, a wide range of Young's modulus of PGSs were prepared with a short curing time as compared to conventional heating.[37] This is because MI activates both primary and secondary alcohol groups of the glycerol molecule. The primary alcohol group is likely to react first with the carboxylic acid and produces a linear structure during the polycondensation process. Interestingly, the microwave energy also enables the secondary alcohol groups to react with the sebacic acids, producing a mixture of linear and branched structures. These highly branched polymers with high number of end groups are especially important since they provide more binding sites for drug molecules and allows tuning of materials to have desirable multifunctionalities for specific interactions.[38] 


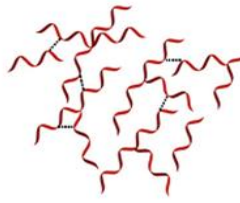

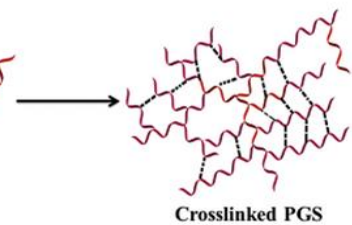

Pre-PGS (MI)

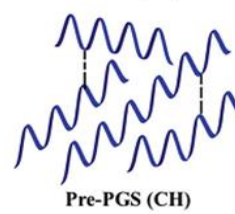
Crosslinked PGS affect degradation rate

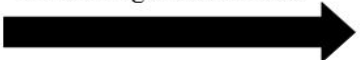

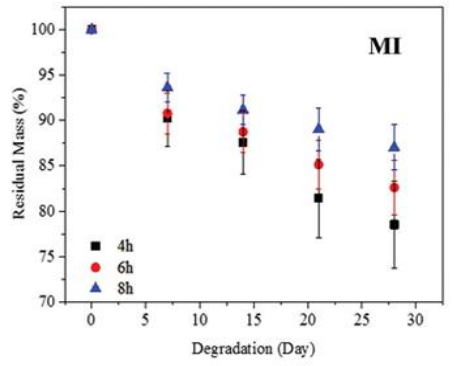

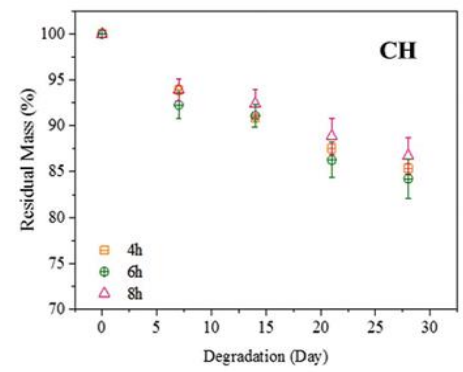

Figure $6 \mathrm{MI}$ varies the molecular structure where highly branched pre-PGS has been produced in the microwave, leading to a wider range of the degradation rates. This figure is adapted with permission from REF [37] , Royal Society Chemistry.

\subsection{Influence of microwave parameters on the polyester properties}

Althouth both single-mode and multi-mode MI systems are more energy-efficient than the conventional heating methods, the microwave intensity in the single-mode is always higher than that in the multi-mode MI.[39-41] Therefore, preparation of pre-PGS is faster in the singlemode MI than in the multi-mode MI when similar degree of esterification is achieved.[37]

The type of solvent used is the primary factor in dictating the interaction between $\mathrm{Ml}$ and reaction medium, specifically the efficiency of converting $\mathrm{MI}$ into heat (referring to Table 1 and Table 2). Higher efficiency enables the reaction medium to achieve the desired temperature within a shorter time. However, in our polycondensation of pre-PGS, a low microwave absorbing medium (i.e. toluene) was delibarately used as a moderate solvent because it could promote the esterification process and its low boiling point could also effectively reduce loss of monomers.

The accuracy of the temperature measurement in the microwave cavity is always crucial. A temperature gradient can be found in a microwave reaction system. For example, the infrared (IR) thermometer used in the CEM Discover LabMate System (CEM Corporation) showed a discrepancy of $15-20^{\circ} \mathrm{C}$ compared to the fiber optic sensor located at the different position in the reaction medium (Figure 7a).[9] The IR measures the temperature by monitoring the IR radiation from the bottom of the reactor. Therefore, using the similar type reactors/vessels is one of the key issue to ensure the reproducibility of $\mathrm{MI}$ experiments. Furthermore, the position of the reactors/vessels in the microwave should be fixed at the same position in the temperature measurement.

In contrast to domestic microwaves, commercial microwave reactors consist of an built-in magnetic stirrer. The stirring process helps to maintain a uniform temperature distribution and mass transfer during the $\mathrm{Ml}$ reaction. $[9,42]$ Figure $7 \mathrm{~b}$ illustrates the difference in the power density in the microwave when liquid is not agitated. Furthermore, the slow stirring rate or no stirring in viscous solvents may create a larger temperature gradient in the reaction medium, which is undesirable for Ml synthesis.[42] Therefore, the reaction medium should be well-mixed to reduce both the temperature gradient and reactants gradient (Figure 7c).

In short, our modified MI approach improves reproducibility by utilising online monitoring of reaction temperature and offers a great degree of control over esterification of pre-polymers. This synthesis pathway can be extended to synthesise other polyester by MI. Importantly, a highly branched polymer could be prepared using $\mathrm{Ml}$ in a short reaction time. These polymers provide more active sites for enhanced drug loading, which have great potential as an improved drug delivery system. 


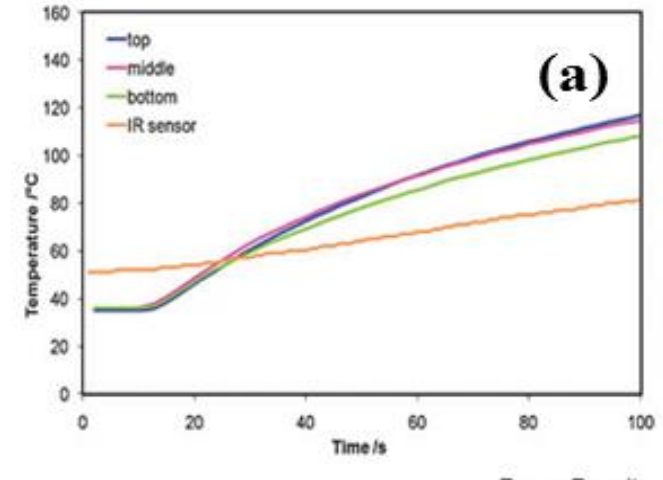

Power Density $\left(\times 10^{7} \mathrm{~W} / \mathrm{m}^{3}\right)$
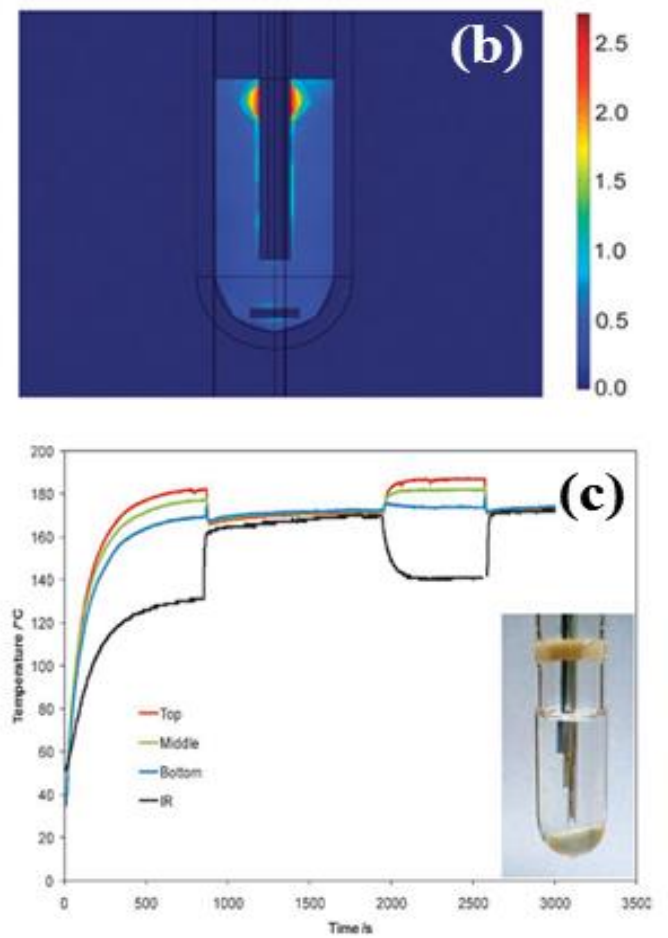

Figure 7 a) Temperature profile of $3 \mathrm{~mL}$ of Methylpyrrolidone (NMP) without stirring in microwave (CEM Discover, 15W constant power). The temperature was measured by the fiber-optic and infrared temperature sensor located at different position. b) Stimulated power density distribution in a $3 \mathrm{~mL}$ of NMP in a quartz vessel. c) Temperature profile of the fiberoptic temperature sensor inside the vessel at different positions (top, middle and bottom) and the infrared temperature sensor located at the bottom of the microwave cavity (below the vessel) when irradiating $3 \mathrm{~mL}$ of NMP (15W, with and without magnetic stirring). Panel $a$ and $b$ is adapted with permission from REF [9], Physical Chemistry Chemical Physics.

\section{Microwave Fluidic System for Synthesis of Nanoparticles}

It is widely acecepted that inorganic nanoparticles, potentially exhibit specular mechanical, electrical, optical, catalytic and magnetic properties due to their small size (the length scale of approximately 1-100 nm). This makes them very useful in a variety of research disciplines.[43-46] Nanoparticles can be synthesized by hydro-/solvo-thermal methods, co-precipitation, sol-gel processing, polymerisation and chemical/physical vapour deposition.[47-51] However, controlled growth of nanoparticles (e.g. particle size and its distribution, shape and morphology, crystallinity and purity and yield) is always very critical in order to realise their full potential in a wide range of applications such as heterogeneous catalysis, photography, information storage, smart electronics, sensing and medicines.

\subsection{Motivation of microwave promoted flow systems}

Flow synthesis, as a potentially controllable and outscaling method, is superior to batch technologies and used as a versatile tool for the synthesis of value-added inorganic $[52,53]$ and organic $[54,55]$ materials. In this section we particularly focus on the preparation of the nanoparticles by a microwave-flow system. Readers are also directed to the recently published complemenetary review articles for the comprehensive use of the microfluidics and conventional heating for the synthesis of organic and inorganic materials.[53, 56, 57]

In principle, microfluidic systems, comprising of small channels $(<1 \mathrm{~mm})$, benefit from large surface-to-volume ratio and provide rapid mixing, controllable mass and heat transfer under continuous flow condition. Further they provide improved control over the size and the polydispersity of particles.[52] Compared to the batch reactors, the synthesis of inorganic nanoparticles in micro-channels has gained considerable attention due to the ease of finely tuning the flow properties.[52]

Yagyu et al have reported the preparation of monodisperse gold nanoparticles in water at room temperature using a low-cost, glass-made microfluidic reactor having a Y-shaped microchannel (Figure 8a).[58] They also discussed the effect of channel width $(260 \mu \mathrm{m}$ and $430 \mu \mathrm{m})$ and flow rates $(0.05,0.06$ and 0.1 $\mathrm{mL} / \mathrm{min}$ ) on the size distribution of the synthesised gold nanoparticles (Figure 8b-h). Their findings revealed that both a slow flow rate $(0.05 \mathrm{~mL} / \mathrm{min})$ and a small channel width $(260 \mu \mathrm{m})$ were essential for the synthesis of monodisperse gold nanoparticles.

Ftouni et al used a microfluidic device to synthesise small diameter citrate-stabilized gold nanoparticles (average diameter $<2 \mathrm{~nm}$ ) by conventional heating at $100{ }^{\circ} \mathrm{C}$ compared to the large diameter gold nanoparticles produced by batch synthesis (Figure 9a).[59] Although the method produced homodispersed $1.8 \mathrm{~nm}$ gold nanoparticles, the amount synthesized was very limited (Figure 9b). 

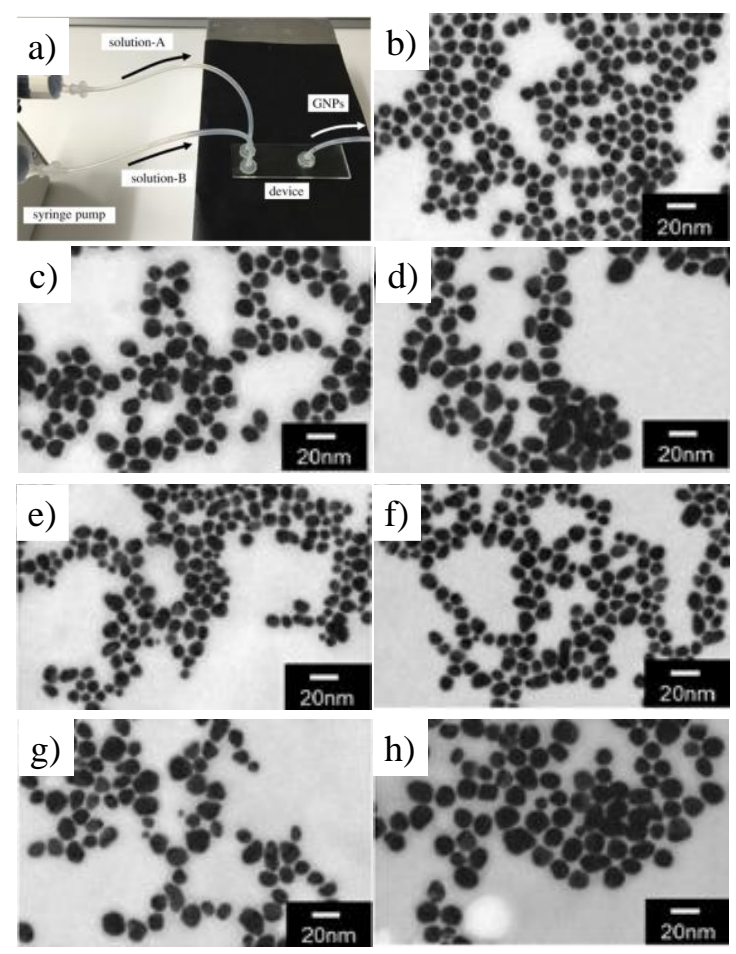

Figure 8 a) Experimental setup for the inorgnaic materials synthesis. TEM images of the synthesised gold nanoparticles with a flow rate of b) $0.05 \mathrm{~mL} / \mathrm{min}, \mathrm{c}$ ) $0.06 \mathrm{~mL} / \mathrm{min}$, d) $0.1 \mathrm{~mL} / \mathrm{min}$ in $260 \mu \mathrm{m}$ width device, e) $0.05 \mathrm{~mL} / \mathrm{min}$, f) $0.06 \mathrm{~mL} / \mathrm{min}, \mathrm{g}) 0.1 \mathrm{~mL} / \mathrm{min}$ in $430 \mu \mathrm{m}$ width device and $h$ ) batch process (100 ml beaker). Panel $a-h$ is adapted with permission from REF. [58], The Institution of Engineering and Technology.

Variations of reaction conditions such as concentration, temperature and reaction time are likely to have a significant effect on both nucleation and particle growth, and a better control over these variables may improve the monodispersity, particle size and its distribution, shape and morphology, crystallinity and purity of the nanoparticles manufactured.[60,61] $\mathrm{MI}$ is believed to havemore homogeneous heating throughout the solution, resulting into more accurate control on nucleation stage. The extra molecular level mixing force generated by microwave is further expected to help mass and energy transfer dramatically, achieving homogeneity throughout the bulk solution.[12, 60-62] However, there are some concerns about the use of MI in the large reactors involving microwave penetration depth, requiring strong microwave intensity, long irradiation time and high pressure resistant reactors. In principal, a capillary reactor ( $<1 \mathrm{~mm}$ diameter) promoted by MI can make use of all advantages of MI but exclude the drawbacks listed for large reactors.

Combining the advantages of the microfluidic reactor with $\mathrm{MI}$, the strong potentials of the method for nanocrystalline materials fabrication can be realised. However, in order to get the full potential of a microwave-flow system with high reproducibility, 1) designing a microwave compatible robust reactor, 2) producing a homogeneous microwave heating and 3) maintaining a good control over the reaction temperature during the chemical reaction are essential. If one of these necessities fails the results are likely not to be reproducible. Thus the researchers are advised to pay particular attention to the reactor design, microwave source and temperature control before designing their experiments.
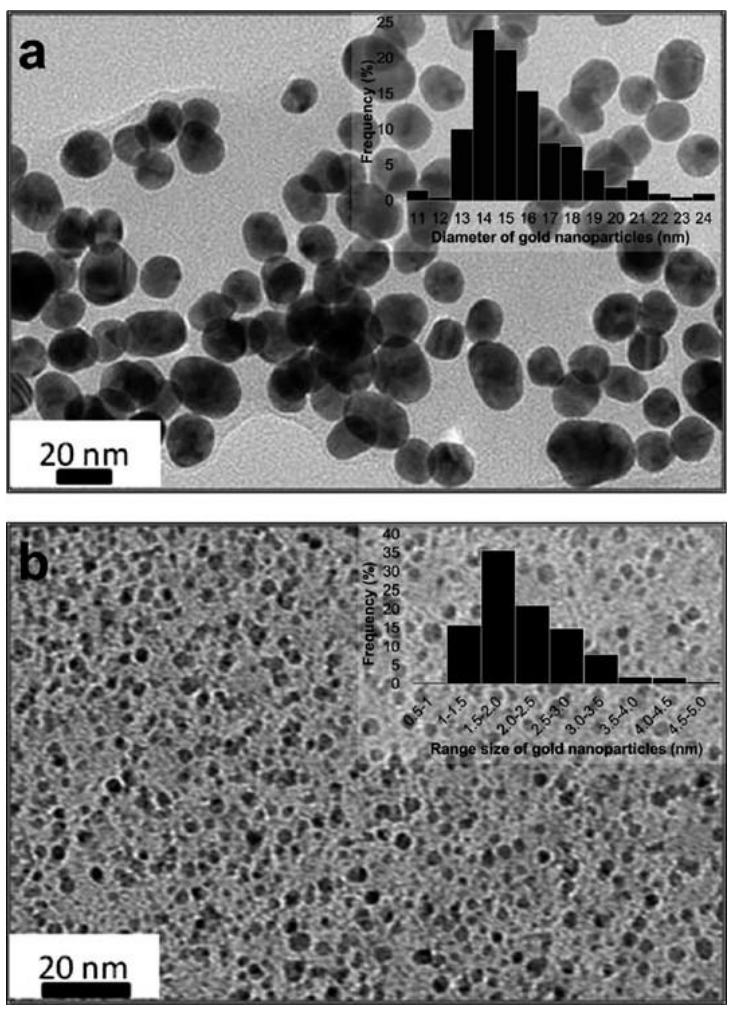

Figure 9 Transmission electron microscopy (TEM) images and size distribution of gold nanoparticles obtained a) in the glassware synthesis and b) in the microfluidic device at a flow rate of $60 \mu \mathrm{L} \mathrm{min}^{-1}$ (residence time $47 \mathrm{~s}$ ), lower panel. Panel $a-b$ is adapted with permission from REF. [59], Royal Society of Chemistry.

For example, Nikam et al recently coupled a single continuous stirred tank reactor (Figure 10a) with a microwave system, and prepared pure nickel nanocrystals (NCs) and the palladium NCs supported by nickel NCs.[63] They used a multimode microwave source (Ragatech Pvt. Ltd., India, 700 W, $2.45 \mathrm{GHz}$ ) without the real-time reaction temperature monitoring neither inside nor outside the microwave zone. The authors characterized the prepared $\mathrm{Pd} / \mathrm{Ni}$ hybrid material using the Powder X-Ray diffraction which showed a peak at $40.1^{\circ}$, attributed to the 111 plane of metallic $\mathrm{Pd}$. The peaks at $44.5^{\circ}, 51.6^{\circ}$, and $76.5^{\circ}$ have been assigned to 111,200 , and 220 planes of $\mathrm{Ni}$ metal (Figure 10b). 

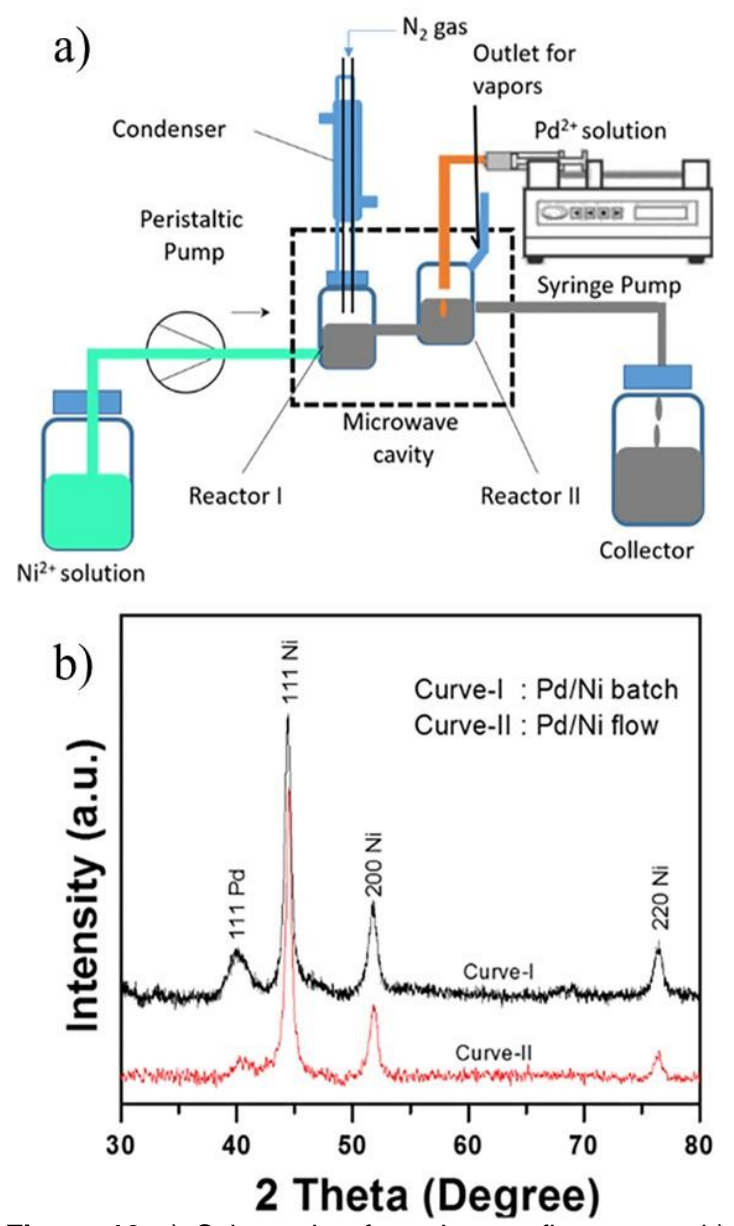

Figure $\mathbf{1 0}$ a) Schematic of continuous flow setup. b) Powder X-ray diffraction pattern for $\mathrm{Pd} / \mathrm{Ni}$ sample prepared in batch and continuous flow method. Panel a$b$ is adapted with permission from REF. [63], American Chemical Society.

\subsection{Experimental design}

Based on the previous reports, we have designed a novel system by coupling microwave heating with continuous flow reactor and online monitoring unit, which was proved to be an attractive route for the rapid, continuous and controlled growth of inorganic metal and metal oxide nanoparticles (Figure 11) .[60, 61]

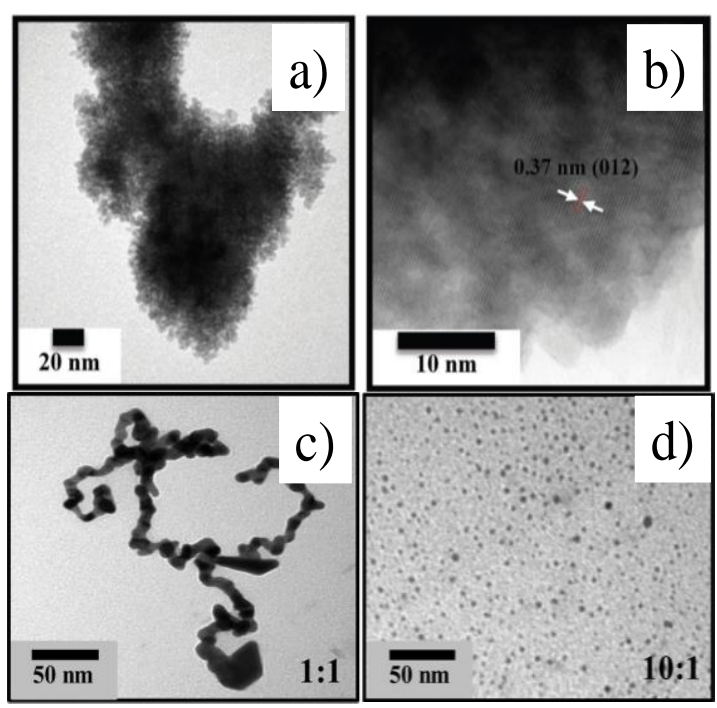

Figure 11 a) TEM image of the microwave-flow synthesized ellipsoid hematite superstructures after mild sonication. b) HRTEM image of ellipsoid hematite superstructures. TEM images of the gold nanoparticles synthesized under constant microwave power of $36 \mathrm{~W}$,

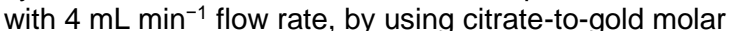
ratios of c) 1 and d) 10. Panel a-b is adapted with permission from REF. [60], Royal Society of Chemistry and Panel c-d is adapted with permission from REF. [61], American Chemical Society.

Briefly, our in-house developed microwave-assisted flow system (Figure 12) is composed of a flow reactor $(6 \mathrm{~mL})$ made of Teflon tubing having 1/8 in. o.d. ( 0.32 $\mathrm{cm})$, a commercially available single mode microwave system (Discover, CEM Microwave Technology Ltd.), a syringe pump (KD Scientific), a thermocouple temperature probe exactly positioned $5 \mathrm{~cm}$ away from the exit of microwave heating zone, and a sample and waste collection unit with backpressure regulator (20 psi, IDEX Health and Science) to control reactor pressure. The whole system works in a harmony to prepare nanoparticles reproducibly. To operate at maximum efficiency the microwave-flow system must be no leaking and completely clean. Often, poor system performance is related to leaks occurring at the connections. 

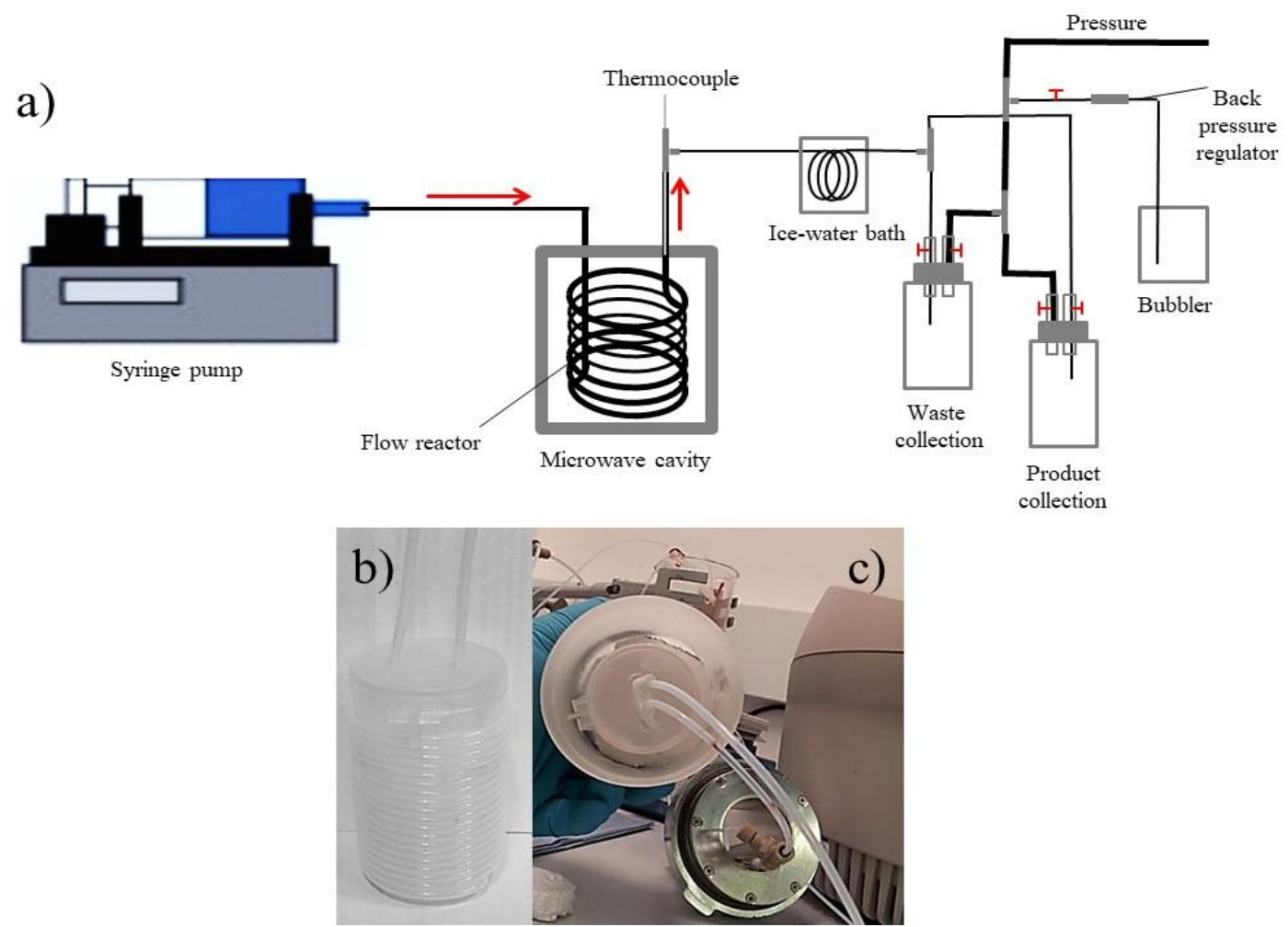

Figure 12 a) Schematic of the microwave-flow system used to prepare hematite[60] and gold nanoparticles[61]. b) Inhouse designed and engineered flow reactor made of Teflon tubing coiled around the polyethylene base for inorganic nanoparticle synthesis. c) A picture of the flow reactor in microwave holder.

As above-mentioned, a microwave transparent (see Table 2) and robust reactor made of low cost materials such as Teflon tubing, should be employed. In our experiments, we engineered our flow reactors by coiling the flexible Teflon tubing around a cylindrical base made of a Teflon or polyethylene material. The size of the reactor and the base is proportional to the size of the microwave cavity. In addition the selection of the base material is totally dependent on the reaction temperature. Basically, the Teflon base can resist the temperature higher than $120^{\circ} \mathrm{C}$ (up to $330^{\circ} \mathrm{C}$ ), while the polyethylene base fits to low temperature reactions. In a microwave-flow system, although the temperature change is closely linked to the main reaction parameters like microwave power, boiling point of the solvent used and the flow rate, it is also highly dependent on the precursor and its concentration dissolved in the reaction solvent. Thus, compared to the pure solvent boiling temperature, one should expect a fast increase in the fluid temperature in micro channels when a solvent/polar precursor mixture was heated by microwave. Figure $\mathbf{1 3}$ shows the temperature change by time in a microwave-flow system at a constant applied microwave power of $30 \mathrm{~W}$ and $2 \mathrm{~mL} / \mathrm{min}$ flow rate. One can easily see that the temperature of pure water reaches $\sim 70{ }^{\circ} \mathrm{C}$ in 5 min while the temperature of $\mathrm{FeCl}_{3}$ solution reaches $\sim 130{ }^{\circ} \mathrm{C}$ under the identical conditions, showing the strong effect of the precursor with $\mathrm{MI}$ and then influencing temperature profile. In addition these results reveal that a critical time is required to reach a stable temperature in the microwave-flow system. One should take into account this time period and will not collect the products untill temperature is being stabilized. Otherwise the collected products will not be homogeneous, instead it can also be a mixture of intermediate and actual products with varying size distribution and morphologies.

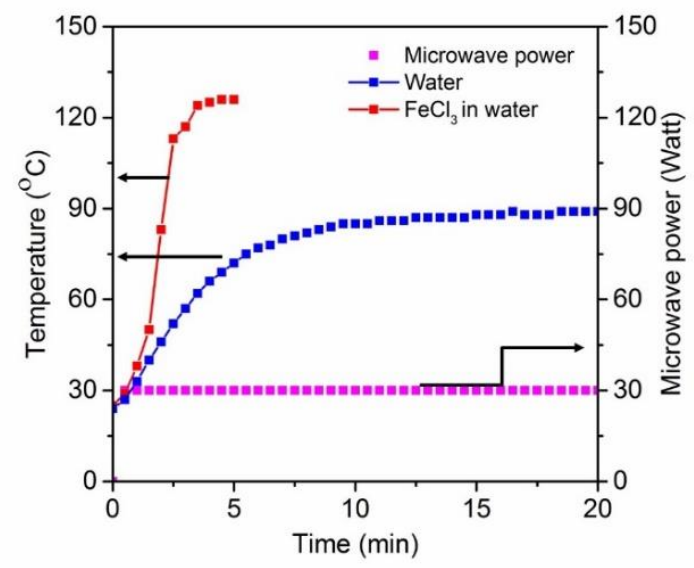

Figure 13 Graph illustrates the change in temperature during microwave irradiation $(30 \mathrm{~W})$ at 1 bar. Flow reactor volume $=6.5 \mathrm{~mL}$, Flow rate $=2 \mathrm{~mL} / \mathrm{min}$ and $\mathrm{FeCl}_{3}$ concentration $=0.1 \mathrm{M}$. 


\subsection{Key factors affecting the nanoparticle synthesis}

In our microwave-flow system, although both synthese processes carried out in an aqueous medium under the same pressure, hematite $\left(\alpha-\mathrm{Fe}_{2} \mathrm{O}_{3}\right)$ synthesis[60] required a reaction temperature above $120^{\circ} \mathrm{C}$, while the gold nanoparticles [61] were prepared at $120^{\circ} \mathrm{C}$. We selected to use the Teflon and polyethylene bases for hematite and gold nanoparticles synthesis, respectively. The readers are therefore advised to pre-evaluate the tangent losses of microwave compatible materials, given in Table 2 before engineering their reactors. $[6,8]$

Microwave sources can be a single-mode or multi-mode. It is wellestablished that single mode $\mathrm{Ml}$ can transfer energy to the reaction medium much more efficiently and faster compared to its multimode counterparts (e.g., domestic microwave systems).[61] However, the main issue for the commercially available singlemode microwaves is the size of their microwave cavity which restricts the size of the designed reactor, as well as the up-scaling of the continuous manufacturing process. Thus designing of single-mode microwave systems offering larger and accessible reaction cavity should be another interest of the microwave flow system which is beyond the topic of the short review.
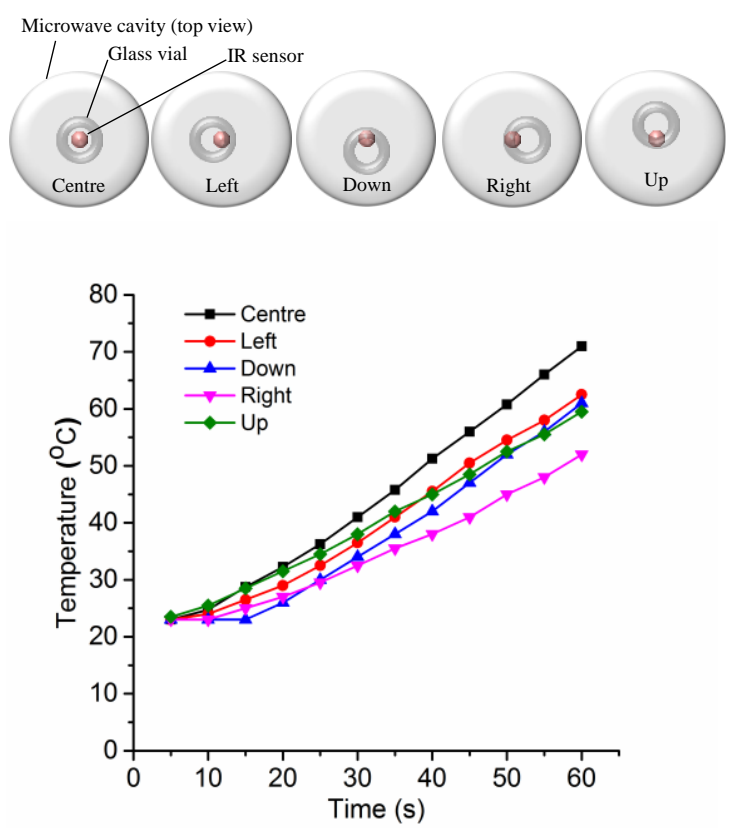

Figure 14 Graph shows the temperature change versus time when a glass vial filled with $10 \mathrm{~mL}$ water was placed in different locations in microwave cavity. (Power $=40 \mathrm{~W}$, Time $=60 \mathrm{~s}$ ) Light grey, dark grey and orange circles refer to microwave cavity, glass vial and IR sensor, respectively.

In order for the reproducible experimental results, as mentioned before another critical point is the position of the flow reactor inside the microwave cavity. Our prelimnary experiments performed by using water as a solvent suggested that the microwave intensity is variable in different locations of the microwave zone (Figure 14). In a typical experiment, a glass vial was filled with $10 \mathrm{~mL}$ water and placed in different locations in the microwave cavity and was irradiated using microwave power of $40 \mathrm{~W}$ for $60 \mathrm{~s}$. Temperature was recorded at every $5 \mathrm{~s}$ using a built-in IR temperature sensor after its calibration. Our findings showed that the flow reactor should be rigid and positioned in the centre of the microwave cavity to reach the highest temperature. Overall the mispositioning of the flow reactor inside the microwave zone is likely to affect the reaction outcomes. Thus some effort should be given to properly position the reactor in the reaction zone.

Finally, the temperature control throughout the microwave-flow process is very essential for the precise control of the produced materials properties. However the current commercially available microwave systems do not allow to real-time monitoring of the temperature distribution in a flow reactor. In our system we have achieved a real-time external temperature monitoring by placing a temperature probe $5 \mathrm{~cm}$ away from the microwave heating zone, and estimated heat loss assuming the forced convection heat transfer in the fluid inside the Teflon reactor and natural convection heat transfer in the surrounding air.[60, 61] However this can not give us temperature distribution radially.

In brief, our previous studies suggest that the key factors affecting the nanoparticle synthesis in the microwave fluidic system are microwave power, precursor concentration and flow rate.[60, 61] In a typical synthesis, these variables can be readily and effectively controlled in the microwave fluidic system benefiting from both single mode microwave heating and flow chemistry. This enables us to reproducibly control the reaction temperature, reactant concentration and reaction time, resulting into much smaller particle size and size distribution. For instance, less crystallised hematite particles were obtained at high precursor concentrations $\left(0.1\right.$ and $\left.0.2 \mathrm{~mol} \mathrm{~L}^{-1}\right)$ while highly crystalline particles were observed at low precursor concentration $(0.05 \mathrm{~mol}$ $\mathrm{L}^{-1}$ ).[60] For gold nanoparticle synthesis, an increase in the flow rate (from $4 \mathrm{~mL} \mathrm{~min}^{-1}$ to $10 \mathrm{~mL} \mathrm{~min}^{-1}$ ) under constant reaction parameters (reactor volume $6 \mathrm{~mL}$, microwave power $36 \mathrm{~W}$, and applied back pressure $20 \mathrm{psi}$ ) resulted in gold nanoparticles composed of high aspect ratio and rod-like particles instead of sphereic gold nanoparticles with well-defined morphology when at $4 \mathrm{~mL} \mathrm{~min}^{-1}$.

\section{Summary and Outlook}

The field of microwave chemistry intensified materials preparation is believed to play a substantial role in future low carbon manufactering due to its higher energy efficency, and potential for preperation of unique nanosized materials and metastable phases with reduced size distribution. In this account, to prepare nanoparticles and polyesters via microwave synthesis have been outlined. In summary, by monitoring and controlling reaction parameters using Ml, properties of the materials can be finetuned to those desirable for their particlular applications (e.g. mechanical properties or biological compatabililty). Furthermore, the reported preparation procedures could be extended to the synthesis of a wide range of functional materials.

The recent advancements in commercial microwave intensified reaction systems, with on-line pressure and temperature measurement, can greatly improve reproducability in comparison to both convention heating methods and retrofitted domestic oven systems. However, accuracy of temperature measurement in microwave reaction systems remains a challenging issue. It can be mediated by placing both fiber and IR temperature detectors in the same position of an idential reactor. 
Continuous development of $\mathrm{MI}$ reaction systems, for example external cooling of reation vessels to maintain a constant temperature (to reduce classical thermal reaction), can further discover potential microwave-induced specific effects, which is an interesting point in establishing the best utilisation of this technique.

Another key step for this cutting edge technology should be it's upscalable material synthesis. The high energy efficiency and reproducability of Ml provide high probability for succesful transition of Ml synthesis route from producing grams to kiligrams.

Continuous flow synthesis systems also have potential to increase reaction efficiency and safety during the preparation of nanomaterials. When coupled with the microwave heating, the continuous microwave-flow systems can be a promising mean for large quantities of useful nanomaterials synthesis. Microwaveassisted multiple flow reactors can allow the out-scaling of the manufacturing process while reducing the total cost. So process intensification can be achievable via homogeneous microwave heating and fine temperature control throughout the chemical reaction. With the current microwave technology, specific focus should also be devoted to sustainable reactor design, the temperature distribution monitoring tools inside the microwave zone and the theoretical modelling of the microwave-flow systems.

\section{Acknowledgements}

C.C. Lau, M.K. Bayazit and P.J.T. Reardon contributed equally to this paper. J. T. acknowledges financial support from Leverhulme Trust (Grant No: RPG-2017-122) and Newton Advanced Fellowship grant (NA150418 and NA170422).

Keywords: microwave, nanoparticle, polymer, batch, flow

\section{References}

1. Gedye, R., et al., The use of microwave ovens for rapid organic synthesis. Tetrahedron Letters, 1986. 27(3): $p$. 279-282.

2. Giguere, R.J., et al., Application of commercial microwave ovens to organic synthesis. Tetrahedron Letters, 1986. 27(41): p. 4945-4948.

3. Estel, L., et al., Continuous flow-microwave reactor Where are we? Chemical Engineering and Processing, 2017. 113: p. 56-64.

4. Opembe, N.N., H. Huang, and S.L. Suib, MicrowaveAssisted Continuous Synthesis of Inorganic Nanomaterials, in Microwaves in Nanoparticle Synthesis. 2013, Wiley-VCH Verlag GmbH \& Co. KGaA. p. 247-269.

5. Hayes, B.L., Microwave Synthesis: Chemistry at the Speed of Light. 2002: CEM Pub.

6. Gabriel, C., et al., Dielectric parameters relevant to microwave dielectric heating. Chemical Society Reviews, 1998. 27(3): p. 213-224.

7. Dallinger, D. and C.O. Kappe, Microwave-Assisted Synthesis in Water as Solvent. Chemical Reviews, 2007. 107(6): p. 2563-2591.

8. Voiry, D., et al., High-quality graphene via microwave reduction of solution-exfoliated graphene oxide. Science, 2016.
9. Robinson, J., et al., Understanding microwave heating effects in single mode type cavities-theory and experiment. Physical Chemistry Chemical Physics, 2010. 12(18): p. 4750-4758.

10. Hoogenboom, R., et al., Microwave-Assisted Chemistry: a Closer Look at Heating Efficiency. Australian Journal of Chemistry, 2009. 62(3): p. 236-243.

11. Feng, S. and R. Xu, New Materials in Hydrothermal Synthesis. Accounts of Chemical Research, 2001. 34(3): p. 239-247.

12. Baghbanzadeh, M., et al., Microwave-Assisted Synthesis of Colloidal Inorganic Nanocrystals. Angewandte Chemie International Edition, 2011. 50(48): p. 11312-11359.

13. de la Hoz, A., A. Diaz-Ortiz, and A. Moreno, Microwaves in organic synthesis. Thermal and non-thermal microwave effects. Chemical Society Reviews, 2005. 34(2): p. 164-178.

14. Wei, K., C. Lai, and Y. Wang, Formation of monetite nanoparticles and nanofibers in reverse micelles. Journal of Materials Science, 2007. 42(14): p. 5340 5346.

15. Wada, Y., et al., Microwave-assisted size control of CdS nanocrystallites. Journal of Materials Chemistry, 2001. 11(7): p. 1936-1940.

16. Jhung, S.H., et al., Microwave Effect in the Fast Synthesis of Microporous Materials: Which Stage Between Nucleation and Crystal Growth is Accelerated by Microwave Irradiation? Chemistry - A European Journal, 2007. 13(16): p. 4410-4417.

17. Wan, A.C.A. and J.Y. Ying, Nanomaterials for in situ cell delivery and tissue regeneration. Advanced Drug Delivery Reviews, 2010. 62(7): p. 731-740.

18. Goldberg, M., R. Langer, and X. Jia, Nanostructured materials for applications in drug delivery and tissue engineering. Journal of Biomaterials Science, Polymer Edition, 2007. 18(3): p. 241-268.

19. Sha, L., et al., Microwave-assisted co-precipitation synthesis of high purity $\beta$-tricalcium phosphate crystalline powders. Materials Chemistry and Physics, 2011. 129(3): p. 1138-1141.

20. Han, J.-K., et al., Synthesis of high purity nano-sized hydroxyapatite powder by microwave-hydrothermal method. Materials Chemistry and Physics, 2006. 99(2): p. 235-239.

21. Reardon, P.J.T., J. Huang, and J. Tang, Mesoporous calcium phosphate bionanomaterials with controlled morphology by an energy-efficient microwave method. Journal of Biomedical Materials Research Part A, 2015. 103(12): p. 3781-3789.

22. Yao, J., et al., Hydroxyapatite nanostructure material derived using cationic surfactant as a template. Journal of Materials Chemistry, 2003. 13(12): p. 3053-3057.

23. Schmidt, S.M., et al., Surfactant based assembly of mesoporous patterned calcium phosphate micron-sized rods. Microporous and Mesoporous Materials, 2006. 94(1): p. 330-338.

24. Vallet-Regí, M., F. Balas, and D. Arcos, Mesoporous Materials for Drug Delivery. Angewandte Chemie International Edition, 2007. 46(40): p. 7548-7558.

25. Reardon, P.J.T., J. Huang, and J. Tang, Morphology Controlled Porous Calcium Phosphate Nanoplates and Nanorods with Enhanced Protein Loading and Release Functionality. Advanced Healthcare Materials, 2013. 2(5): p. 682-686. 
26. Ching Lau, C., et al., Phase-Tunable Calcium Phosphate Biomaterials Synthesis and Application in Protein Delivery. ACS Biomaterials Science \& Engineering, 2015. 1(10): p. 947-954.

27. Thomas Reardon, P.J., et al., Dimensionally and compositionally controlled growth of calcium phosphate nanowires for bone tissue regeneration. Journal of Materials Chemistry B, 2013. 1(44): p. 6170-6176. http://pubs.rsc.org/en/content/articlehtml/2013/tb/c3tb2 1073a\#cit29

28. Peng, Z.A. and X. Peng, Mechanisms of the Shape Evolution of CdSe Nanocrystals. Journal of the American Chemical Society, 2001. 123(7): p. 13891395.

29. Lerner, E., R. Azoury, and S. Sarig, Rapid precipitation of apatite from ethanol-water solution. Journal of Crystal Growth, 1989. 97(3): p. 725-730.

30. Yu, S.-H., et al., A Novel Solventothermal Synthetic Route to Nanocrystalline $\operatorname{CdE}(E=S$, Se, Te) and Morphological Control. Chemistry of Materials, 1998. 10(9): p. 2309-2312.

31. Oliver Kappe, C., Microwave dielectric heating in synthetic organic chemistry. Chemical Society Reviews, 2008. 37(6): p. 1127-1139.

32. Wiesbrock, F., R. Hoogenboom, and U.S. Schubert, Microwave-Assisted Polymer Synthesis: State-of-theArt and Future Perspectives. Macromolecular Rapid Communications, 2004. 25(20): p. 1739-1764.

33. Nagahata, R., et al., Microwave-Assisted Single-Step Synthesis of Poly(lactic acid) by Direct Polycondensation of Lactic Acid. Macromolecular Rapid Communications, 2007. 28(4): p. 437-442.

34. Kéki, S., et al., Fast Microwave-Mediated Bulk Polycondensation of d,I-Lactic Acid. Macromolecular Rapid Communications, 2001. 22(13): p. 1063-1065.

35. Aydin, H.M., et al., Microwave-assisted rapid synthesis of poly(glycerol-sebacate) elastomers. Biomaterials Science, 2013. 1(5): p. 503-509.

36. Li, X., et al., Criteria for Quick and Consistent Synthesis of Poly(glycerol sebacate) for Tailored Mechanical Properties. Biomacromolecules, 2015. 16(5): p. 15251533.

37. Lau, C.C., et al., Tailoring degree of esterification and branching of poly(glycerol sebacate) by energy efficient microwave irradiation. Polymer Chemistry, 2017. 8(26): p. 3937-3947.

38. Voit, B.I. and A. Lederer, Hyperbranched and Highly Branched Polymer Architectures-Synthetic Strategies and Major Characterization Aspects. Chemical Reviews, 2009. 109(11): p. 5924-5973.

39. Barlow, S. and S.R. Marder, Single-mode microwave synthesis in organic materials chemistry. Advanced Functional Materials, 2003. 13(7): p. 517-+.

40. Kuhnert, N., Microwave-Assisted Reactions in Organic Synthesis-Are There Any Nonthermal Microwave Effects? Angewandte Chemie International Edition, 2002. 41(11): p. 1863-1866.

41. Stadler, A., et al., Scalability of Microwave-Assisted Organic Synthesis. From Single-Mode to Multimode Parallel Batch Reactors. Organic Process Research \& Development, 2003. 7(5): p. 707-716.

42. Herrero, M.A., J.M. Kremsner, and C.O. Kappe, Nonthermal Microwave Effects Revisited: On the Importance of Internal Temperature Monitoring and Agitation in Microwave Chemistry. The Journal of Organic Chemistry, 2008. 73(1): p. 36-47.
43. Kango, S., et al., Surface modification of inorganic nanoparticles for development of organic-inorganic nanocomposites- $A$ review. Progress in Polymer Science, 2013. 38(8): p. 1232-1261.

44. Lemire, J.A., J.J. Harrison, and R.J. Turner, Antimicrobial activity of metals: mechanisms, molecular targets and applications. Nature Reviews Microbiology, 2013. 11(6): p. 371-384.

45. Wang, H.L. and H.J. Dai, Strongly coupled inorganicnano-carbon hybrid materials for energy storage. Chemical Society Reviews, 2013. 42(7): p. 3088-3113.

46. Zhu, Y.J. and F. Chen, Microwave-Assisted Preparation of Inorganic Nanostructures in Liquid Phase. Chemical Reviews, 2014. 114(12): p. 6462-6555.

47. Cushing, B.L., V.L. Kolesnichenko, and C.J. O'Connor, Recent advances in the liquid-phase syntheses of inorganic nanoparticles. Chemical Reviews, 2004. 104(9): p. 3893-3946.

48. Kammler, H.K., L. Madler, and S.E. Pratsinis, Flame synthesis of nanoparticles. Chemical Engineering \& Technology, 2001. 24(6): p. 583-596.

49. Kruis, F.E., H. Fissan, and A. Peled, Synthesis of nanoparticles in the gas phase for electronic, optical and magnetic applications - $A$ review. Journal of Aerosol Science, 1998. 29(5-6): p. 511-535.

50. Swihart, M.T., Vapor-phase synthesis of nanoparticles. Current Opinion in Colloid \& Interface Science, 2003. 8(1): p. 127-133.

51. Xu, H.X., B.W. Zeiger, and K.S. Suslick, Sonochemical synthesis of nanomaterials. Chemical Society Reviews, 2013. 42(7): p. 2555-2567.

52. Abou-Hassan, A., O. Sandre, and V. Cabuil, Microfluidics in Inorganic Chemistry. Angewandte Chemie International Edition, 2010. 49(36): p. 62686286.

53. Zhang, J.Y., et al., Continuous flow chemistry: New strategies for preparative inorganic chemistry. Coordination Chemistry Reviews, 2016. 324: p. 39-53.

54. Rinaldi, L., et al., A Microwave-Based Chemical Factory in the Lab: From Milligram to Multigram Preparations. Journal of Chemistry, 2015.

55. Gutmann, B., D. Cantillo, and C.O. Kappe, ContinuousFlow TechnologyA Tool for the Safe Manufacturing of Active Pharmaceutical Ingredients. Angewandte Chemie-International Edition, 2015. 54(23): p. 66886728.

56. Hou, X., et al., Interplay between materials and microfluidics. Nature Reviews Materials, 2017. 2(5).

57. Movsisyan, M., et al., Taming hazardous chemistry by continuous flow technology. Chemical Society Reviews, 2016. 45(18): p. 4892-4928.

58. Yagyu, H., et al., Continuous flow synthesis of monodisperse gold nanoparticles by liquid-phase reduction method on glass microfluidic device. Micro \& Nano Letters, 2017. 12(8): p. 536-539.

59. Ftouni, J., et al., Highly controlled synthesis of nanometric gold particles by citrate reduction using the short mixing, heating and quenching times achievable in a microfluidic device. Nanoscale, 2012. 4(15): p. 4450-4454

60. Bayazit, M.K., et al., A microwave promoted continuous flow approach to self-assembled hierarchical hematite superstructures. Green Chemistry, 2016. 18(10): p. 3057-3065

61. Bayazit, M.K., et al., Controllable Synthesis of Gold Nanoparticles in Aqueous Solution by Microwave 
Assisted Flow Chemistry. Acs Sustainable Chemistry \& Engineering, 2016. 4(12): p. 6435-6442.

62. Horikoshi, S., et al., Access to small size distributions of nanoparticles by microwave-assisted synthesis. Formation of $\mathrm{Ag}$ nanoparticles in aqueous carboxymethylcellulose solutions in batch and continuous-flow reactors. Nanoscale, 2010. 2(8): p. 1441-1447.

63. Nikam, A.V., A.A. Kulkarni, and B.L.V. Prasad, Microwave-Assisted Batch and Continuous Flow Synthesis of Palladium Supported on Magnetic Nickel Nanocrystals and Their Evaluation as Reusable Catalyst. Crystal Growth \& Design, 2017. 17(10): p. 5163-5169. 


\section{Entry for the Table of Contents}

\section{PERSONAL ACCOUNT}

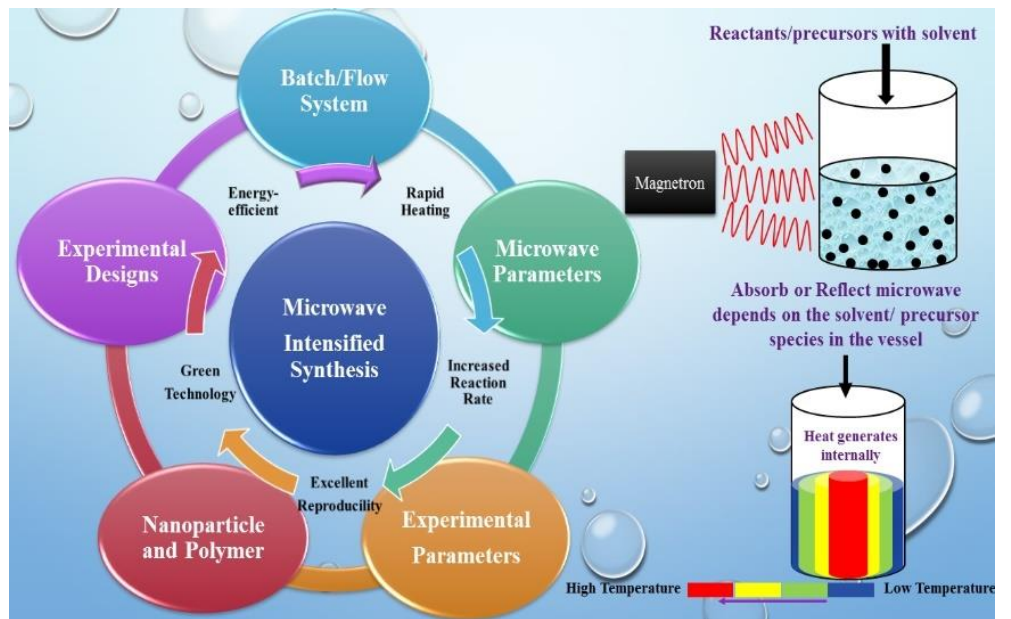

We describe a guideline on preparing the nanoparticles and polyester using the microwave intensified chemical approach, in both a batch and a fluidic system. In particular, the preparation of the nanoparticles (i.e. calcium phosphate, gold and hematite) and polyester (i.e. poly(glycerol sebacate)) have been explored in details. The microwave parameters play significant roles not only in reaction rates, but also in the material properties (e.g. morphology, phase, molecular structure and particle size). The fundamental underlying the microwave chemistry including the interaction of the microwave with the solvent species and reactor has also been discussed with some clues to overcome the challenges facing.
Chi Ching Lau, Mustafa Kemal Bayazit, Philip James Thomas Reardon, Junwang Tang*

Page No. - Page No.

Microwave Intensified Synthesis: Batch and Flow Chemistry 